\title{
Nano-scale processes behind ion-beam cancer therapy ${ }^{\star}$
}

\author{
Eugene Surdutovich ${ }^{1}$, Gustavo Garcia ${ }^{2}$, Nigel Mason ${ }^{3}$, and Andrey V. Solov'yov ${ }^{4, a}$ \\ 1 Department of Physics, Oakland University, Rochester, Michigan 48309, USA \\ 2 Instituto de Fisica Fundamental, CSIC, Serrano 113-bis, 28006 Madrid, Spain \\ 3 Deptartment of Physical Sciences, The Open University, Walton Hall, Milton Keynes, MK7 6AA, UK \\ 4 MBN Research Center, Altenhoferallee 3, 60438 Frankfurt am Main, Germany
}

Received 1 March 2016

Published online 14 April 2016 - (c) EDP Sciences, Società Italiana di Fisica, Springer-Verlag 2016

\begin{abstract}
This topical issue collates a series of papers based on new data reported at the third Nano-IBCT Conference of the COST Action MP1002: Nanoscale Insights into Ion Beam Cancer Therapy, held in Boppard, Germany, from October 27th to October 31st, 2014. The Nano-IBCT COST Action was launched in December 2010 and brought together more than 300 experts from different disciplines (physics, chemistry, biology) with specialists in radiation damage of biological matter from hadron-therapy centres, and medical institutions. This meeting followed the first and the second conferences of the Action held in October 2011 in Caen, France and in May 2013 in Sopot, Poland respectively. This conference series provided a focus for the European research community and has highlighted the pioneering research into the fundamental processes underpinning ion beam cancer therapy.
\end{abstract}

\section{Introduction}

Ion beam cancer therapy (IBCT, or hadron therapy) represents an effective method for providing high-dose delivery into tumours, thereby maximizing the probability of killing the cancer cells whilst simultaneously minimizing the radiation damage to surrounding healthy tissue [1-3]. Despite its high cost, proton-beam therapy is widely spread around the world with over 60 operational centres $^{1}$. In ten European and Asian centres, patients are irradiated with carbon ions. Nonetheless, the full potential of these therapies can only be realised by achieving a better understanding of physical, chemical and biological mechanisms, over a range of time and space scales, that leads to cell inactivation under ion radiation.

The damaging effect of ionizing radiation has been known for many years. It has been commonly accepted that high-energy tracks formed by $\alpha, \beta$, and $\gamma$ radiation and atomic ions ionize cell components along the track, thereby leading to various dissociation channels and to the formation of damaging radicals. This has led to intensive research on the study of the mechanisms for the formation of such radicals and the fragmentation pattern of biomolecules by photons, electrons and ions. Such fundamental data underpins the study of radiation protec-

\footnotetext{
* Contribution to the Topical Issue "COST Action Nano-IBCT: Nano-scale Processes Behind Ion-Beam Cancer Therapy", edited by Andrey V. Solov'yov, Nigel Mason, Gustavo Garcia and Eugene Surdutovich.

a e-mail: solovyov@mbnresearch.com

1 As of February $2016^{2}$.
}

tion and the development of biomedical uses of different radiation, generally called radiotherapy, for treatment, of tumoural diseases in particular. The next generation of radiotherapy may be based on hadron therapy ${ }^{2}$ and in particular ion-beam therapy. To date the development of ion beam therapy has been based on empirical rather than phenomenological or ab initio scientific methods [4]. The emergence of the "RADAM" [4] and then "NanoIBCT" 3 communities has played an important role in attracting physicists, chemists, and biologists into the field to tackle a plethora of scientific questions raised by the technological advances in this field.

The majority of biological effects of ion beams are associated with the process of ionization of the medium by traversing ions. It is commonly accepted that secondary electrons, ejected by ionization, are mainly responsible for DNA damage, either breaking the DNA strands directly, or reacting with molecules of tissue, producing free radicals and other DNA reactive species. Macroscopically, the advantages of using ion beams compared to photons are related to the presence of a Bragg peak in the depth-dose distribution, where the production of secondary electrons is maximized. This localizes irradiation effects deep in tissue thus increasing the treatment efficiency and reducing side effects by sparing neighboring healthy tissue. However, the mechanisms involved in radiation damage on the

\footnotetext{
2 Particle therapy co-operative group, http://www.ptcog. $\mathrm{ch} /$ index.php/facilities-in-operation (accessed on 06/2014).

${ }^{3}$ Cost action nano-IBCT, http://mbnresearch.com/ project-nanoibct (accessed on 02/2016).
} 
nanoscale and molecular level are still a subject of fundamental multidisciplinary research.

In 2010-2014, the European Concerted Research Action, COST Action MP1002: "Nano-scale insights in ion beam cancer therapy (Nano-IBCT)" was devoted to acquiring a deeper understanding of radiation induced damage with ions on the nanoscopic and molecular level. This endeavour clustered around the multiscale approach to the physics of radiation damage with ions $[1,5]$, designed to achieve a quantitative understanding of the physical, chemical, and biological effects that take place on a wide range of spatial, temporal, and energy scales. The COST Action combined European experimental and theoretical expertise in several topics including nuclear reactions and electromagnetic processes during the propagation of ion beams in tissue, primary ionization in the medium (water and biological molecules), direct damage and production of secondary species (secondary electrons, radicals, holes), propagation of secondary species and their interaction with DNA, and radiobiological scale effects.

Action was formally launched in December 2010 and since then has brought together more than 300 experts from different disciplines (physics, chemistry, biology, etc.) drawn from more than fifty different institutions including hadron therapy centres and medical institutions. The Action also engaged with colleagues working in countries outside the EU, including Canada, Australia, Japan, India, China and the USA. Two thirds of those participating were early career researches and a quarter were postgraduate students half of which were young female researchers. Within the framework of the COST Action Nano-IBCT three major conferences (held in Caen, France October 2011, Sopot, Poland May 2013 and Boppard, Germany 2013) and 12 workshops were organised. The Action also supported more than 100 Short Term Scientific missions between different institutions and countries, which resulted in more nearly 200 publications in high impact journals. For further details, see the Action's website ${ }^{3}$. The 3rd Nano-IBCT conference, held in Boppard, Germany, October 27-31, 2014, provided the opportunity to review recent progress in the field of radiation damage to biomolecular systems and how such knowledge can be applied to the development of new cancer therapies.

The main objective of the Action was to address the basic scientific questions which underpin the nanoscopic and molecular mechanisms associated with Ion Beam Cancer Therapy. In particular, the following goals were pursued:

- Understanding the unique features of ion irradiation on the molecular level, e.g. site and bond selectivity, clustered damage, local temperature and chemical effects. Some of the open questions are related to the ratio of direct/indirect damage, the mechanisms leading to double strand break (single or multiple electron induced fragmentation) and the elucidation of possible lethal effects that are not present during photon irradiation.

- To establish comprehensive databases of recommended values for all the major processes involved in IBCT: ion and electron interaction cross sections, energy loss in biologically relevant systems, etc. This objective implies an important experimental and theoretical effort to determine differential and integral cross sections, both elastic and inelastic, for low incident energies.

- To yield a quantitative prediction of dose distribution and molecular damage generated by the passage of an ion beam through cells, for example determining the rate and type of initial double strand breaks.

- To develop a multiscale code for the quantitative analysis of radiobiological effects and therapy planning, tested at different levels with experiments; also including reliable estimates of the relative biological effectiveness (RBE) for different ions.

- To develop a new low energy particle track simulation method based on the distribution functions derived from evaluated experimental and theoretical cross sectional data and energy loss providing information on energy distribution and induced damage at the molecular level.

The COST Action was organised through five Working Groups (WGs) each dedicated to a specific topic. WG1: Ion Propagation, WG2: Primary ionization in the medium, direct damage and production of secondary species, WG3: Propagation of secondary species, WG4: Electron attack on DNA, WG5: Radiobiological scale effects. These working groups form the pillars upon which the research plan for a multiscale approach to the physics of ion-beam cancer therapy are based [1]. Below, we briefly overview the work of the Nano-IBCT WGs with the emphasis of some of the results published in this topical issue.

The multiscale approach to the physics of radiation damage has substantially advanced and extended since 2010 and in its most complete and consistent form is presented in this topical issue. On the scale of electromagnetic interactions, the effect of the presence of biomolecules in the medium on ionization cross sections, stopping power, dose deposition in a cell was explored [6,7]. Another advance on this scale was the analysis of secondary electron production by nanoparticles $[8,9]$. The radial dose deposition as well as transport of secondary particles on the scales pertinent to this transport have also been investigated [10-12]. Considerable progress has been achieved in the analysis of the shock waves on a nanometre scale consequent to energy relaxation in the medium, predicted in reference [13]. Thermomechanical effects of these shock waves on the radiation damage have been studied in references $[14,15]$. Several works were devoted to the DNA damage mechanisms, for example clustered damage [16] and double ionization events [17]. Finally, the multiscale approach has been synthesised in reference [1], where the damage probability curves were obtained for plasmid DNA and a 'recipe' for the calculation of the survival curves for real cells was suggested. Thus the Action's research explored physical and chemical process from the time of the ion entering the tissue to the cell's inactivation, however much more needs to be done before this knowledge can be exploited in clinical procedures. 
The "Ion Propagation (Nuclear reactions and electromagnetic processes)" working group was responsible for the accurate nuclear, atomic physics, and electrodynamics computations. This topic is not presented specifically in this volume, but there were a number of publications in the previous topical issue [18], such as references [19-21], and others. Primary ionization in the medium (water and biological molecules), direct damage and production of secondary species were the scope of the second work group. The paper by Boscolo et al. [22] is devoted to the calculation of the efficiency of thermoluminescent dosimeters. The paper of Hilgers et al. [23] presents the results of a simulation of an ion-counting nanodosimeter. Theoretical studies of ionization of biomolecules with protons are reported by Paredes et al. [24]. Finally, a theoretical analysis of secondary electron production in liquid water sensitized with carbon nanoparticles is reprorted by Verkhovtsev et al. [9]. These works follow the previous volume's papers [25-27] and others.

Propagation of secondary species (secondary electrons, radicals, holes) was the focus of the third working group and its activity is presented by the following papers. The paper by Sushko et al. [28] demonstrates the new capabilities of the MBN Explorer software, developed at the MBN Research Center, in applications to chemically reactive systems. Alexander et al. [29] carried out energydependent track structure parametrisation for proton and carbon ions based on nanometric simulations. Surdutovich and Solov'yov [11] have studied transport of secondary particles analytically and presented a more detailed radial dose distribution. More track structure simulations are reported by Arce et al. [30] and a simulation of energy deposition by secondary electrons is reported by Dapor et al. [31]. The propagation of secondary species was represented in the previous topical issue as well [32,33], etc.

Electron attack on DNA (dissociative electron attachment and direct ionization) was the topic of the fourth work group and its achievements are presented by the following papers. The paper of Piatnytskyi et al. [34] studies the action of hydrogen peroxide on DNA. Rudek et al. [35] report experimental studies of DNA constituents fragmentation on proton impact. Kumar et al. [36] compare and discuss experimental studies of DNA irradiated with electrons. Theoretical studies of electron attachment are reported in by Lacombe et al. [37] van der Burgt et al. [38] experimentally studied electron impact fragmentation of adenine. A study of dissociative electron attachment to sulfur containing compounds is presented by Kopyra and Władziński [39]. A theoretical study of dissociative electron attachment to gas-phase uracil and pyrimidine molecules is reported in the paper by Carelli et al. [40]. Ionization of pyrimidine with electrons and protons is studied theoretically by Champion et al. [41]. Bacchus-Montabonel [42] reported on the conformation and orientation dependence in ion-induced collisions with DNA and RNA building blocks. Finally, sensitizing effects of $\mathrm{Pt}$ and $\mathrm{Br}$ on DNA damage is studied by Śmiałek et al. [43]. The previous volume's contributions on related topics include references $[26,44-51]$.
Radiobiological scale effects (DNA DSBs detection, prediction and cellular consequences) were the focus of the fifth working group. The papers devoted to these topics are not in this issue because this is a physics journal, while the effects are biological. The previous volume, however, featured references $[52,53]$.

Concluding, the COST Action Nano-IBCT played a very important role in the foundation of a strong European Nano-IBCT community, which inherited and broadened the traditions of the initial RADAM network. Ideas emerging during the Nano-IBCT COST Action led to many research collaborations including the establishment of the current ARGENT programme exploring nanoparticles as radiosenistizers (www.argent.eu). A more detailed review of the research of ion beam therapy drawing on the results of the Nano-IBCT Acost action will appear in the form of a book to be published by Springer in 2016 .

We are grateful to the support of COST Action MP1002 "Nano-scale insights in ion beam cancer therapy" and FP7 ITN-ARGENT.

\section{References}

1. E. Surdutovich, A. Solov'yov, Eur. Phys. J. D 68, 353 (2014)

2. D. Schardt, T. Elsässer, D. Schulz-Ertner, Rev. Mod. Phys. 82, 383 (2010)

3. M. Durante, J. Loeffler, Nat. Rev. Clin. Oncol. 7, 37 (2010)

4. I. Baccarelli, F. Gianturco, E. Scifoni, A. Solov'yov, E. Surdutovich, Eur. Phys. J. D 60, 1 (2010)

5. A. Solov'yov, E. Surdutovich, E. Scifoni, I. Mishustin, W. Greiner, Phys. Rev. E 79, 011909 (2009)

6. P. de Vera, R. Garcia-Molina, I. Abril, A.V. Solov'yov, Phys. Rev. Lett. 110, 148104 (2013)

7. P. de Vera, E. Surdutovich, I. Abril, R. Garcia-Molina, A.V. Solov'yov, Eur. Phys. J. D 68, 96 (2014)

8. A.V. Verkhovtsev, A.V. Korol, A.V. Solov'yov, Phys. Rev. Lett. 114, 063401 (2015)

9. A. Verkhovtsev, S. McKinnon, P. de Vera, E. Surdutovich, S. Guatelli, A. Korol, A. Rosenfeld, A.V. Solov'yov, Eur. Phys. J. D 69, 116 (2015)

10. E. Surdutovich, A.V. Solov'yov, Eur. Phys. J. D 66, 245 (2012)

11. E. Surdutovich, A.V. Solov'yov, Eur. Phys. J. D 69, 193 (2015)

12. M. Bug, E. Surdutovich, H. Rabus, A.B. Rosenfeld, A.V. Solov'yov, Eur. Phys. J. D 66, 291 (2012)

13. E. Surdutovich, A. Solov'yov, Phys. Rev. E 82, 051915 (2010)

14. A.V. Yakubovich, E. Surdutovich, A.V. Solov'yov, Nucl. Instrum. Meth. B 279, 135 (2012)

15. E. Surdutovich, A.V. Yakubovich, A.V. Solov'yov, Sci. Rep. 3, 1289 (2013)

16. E. Surdutovich, D.C. Gallagher, A.V. Solov'yov, Phys. Rev. E 84, 051918 (2011)

17. E. Surdutovich, A.V. Solov'yov, Eur. Phys. J. D 66, 206 (2012)

18. M. Śmiałek, P. Limão-Vieira, N. Mason, A. Solovyov, Eur. Phys. J. D 68, 312 (2014) 
19. R. Delaunay, et al., Eur. Phys. J. D 68, 162 (2014)

20. M. Bug, G. Hilgers, W. Baek, H. Rabus, Eur. Phys. J. D 68, 217 (2014)

21. C. Champion, M. Quinto, M. Bug, W. Baek, P. Weck, Eur. Phys. J. D 68, 205 (2014)

22. D. Boscolo, E. Scifoni, A. Carlino, C.L. Tessa, T. Berger, M. Durante, V. Rosso, M. Kraemer, Eur. Phys. J. D 69, $286(2015)$

23. G. Hilgers, M. Bug, E. Gargioni, H. Rabus, Eur. Phys. J. D 69, $239(2015)$

24. S. Paredes, C. Illescas, L. Méndez, Eur. Phys. J. D 69, 178 (2015)

25. S. Greilich, U. Hahn, M. Kiderlen, C. Andersen, N. Bassler, Eur. Phys. J. D 68, 327 (2014)

26. P. van der Burgt, Eur. Phys. J. D 68, 135 (2014)

27. M. Fuss, A. Sanz, F. Blanco, P. Limão Vieira, M. Brunger, G. García, Eur. Phys. J. D 68, 161 (2014)

28. G. Sushko, I. Solov'yov, A. Verkhovtsev, S. Volkov, A. Solov'yov, Eur. Phys. J. D 70, 12 (2016)

29. F. Alexander, C. Villagrasa, H. Rabus, J. Wilkens, Eur. Phys. J. D 69, 216 (2015)

30. P. Arce, A. Muñoz, M. Moraleda, J.G. Ros, F. Blanco, J. Perez, G. García, Eur. Phys. J. D 69, 188 (2015)

31. M. Dapor, I. Abril, P. de Vera, R. Garcia-Molina, Eur. Phys. J. D 69, 165 (2015)

32. X. Han, W. Cantrell, E. Escobar, S. Ptasinska, Eur. Phys. J. D 68, 46 (2014)

33. B. Bahnev, M. Bowden, A. Stypczyńska, S. Ptasińska N. Mason, N. Braithwaite, Eur. Phys. J. D 68, 140 (2014)

34. D. Piatnytskyi, O. Zdorevskyi, S. Perepelytsya, S. Volkov, Eur. Phys. J. D 69, 255 (2015)

35. B. Rudek, A. Arndt, D. Bennett, M. Wang, H. Rabus, Eur. Phys. J. D 69, 237 (2015)
36. S. Kumar, M. Murali, P. Kushwaha, Eur. Phys. J. D 69, $204(2015)$

37. L. Lacombe, P. Dinh, P.G. Reinhard, E. Suraud, L. Sanche, Eur. Phys. J. D 69, 195 (2015)

38. P. van der Burgt, S. Finnegan, S. Eden, Eur. Phys. J. D 69, $173(2015)$

39. J. Kopyra, J. Władziński, Eur. Phys. J. D 69, 157 (2015)

40. F. Carelli, F. Gianturco, J. Franz, M. Satta, Eur. Phys. J. D 69, $143(2015)$

41. C. Champion, M. Quinto, P. Weck, Eur. Phys. J. D 69, $127(2015)$

42. M.C. Bacchus-Montabonel, Eur. Phys. J. D 69, 107 (2015)

43. M. Śmiałek, S. Ptasińska, J. Gow, S. Hoffmann, N. Mason, Eur. Phys. J. D 69, 121 (2015)

44. A. Stypczyńska, T. Nixon, N. Mason, Eur. Phys. J. D 68, $333(2014)$

45. J. Franz, F. Gianturco, Eur. Phys. J. D 68, 279 (2014)

46. M.C. Castrovilli, P. Bolognesi, A. Casavola, A. Cartoni, D. Catone, P. O'Keeffe, L. Avaldi, Eur. Phys. J. D 68, 253 (2014)

47. V. Štěpán, M. Davídková, Eur. Phys. J. D 68, 240 (2014)

48. A. Piekarczyk, I. Bald, H. Flosadóttir, B. Ómarsson, A. Lafosse, O. Ingólfsson, Eur. Phys. J. D 68, 146 (2014)

49. A. Yakubovich, A. Solov'yov, Eur. Phys. J. D 68, 145 (2014)

50. E. Alizadeh, L. Sanche, Eur. Phys. J. D 68, 97 (2014)

51. M. Śmiałek, S. Ptasińska, J. Gow, C.D. Pieve, N. Mason, Eur. Phys. J. D 68, 85 (2014)

52. A. Kowalska, K. Czerski, M. Kaczmarski, M. Lewocki, B. Masojć, A. Łukowiak, Eur. Phys. J. D 69, 79 (2015)

53. J. Deperas-Standylo, E. Gudowska-Nowak, S. Ritter, Eur. Phys. J. D 68, 204 (2014) 